\title{
Cytokine Release Syndrome
}

National Cancer Institute

\section{Source}

National Cancer Institute. Cytokine Release Syndrome. NCI Thesaurus. Code C78251.

A syndrome that occurs after therapeutic infusion of antibodies into the blood and is characterized by nausea, headache, tachycardia, hypotension, rash, and shortness of breath. It is caused by the release of cytokines from the cells that are targeted by the antibodies. Most patients experience a mild to moderate reaction; however, the reaction may be severe and life-threatening. 